\title{
Lesões nos Jogadores de Futebol Profissional do Marília Atlético Clube: Estudo de Coorte Histórico do Campeonato Brasileiro de 2003 a 2005*
}

\author{
Injuries in the Professional Soccer Players of Marília Atlético Clube: \\ a Cohort Study of the Brazilian Championship, 2003 to 2005
}

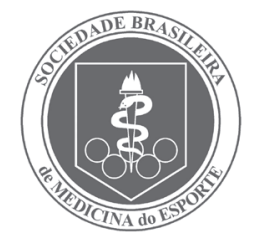

Artigo Original
Evandro Pereira Palacio Bruno Moreira Candeloro Aline de Almeida Lopes

*.Serviço de Ortopedia e Traumatologia da Faculdade de Medicina de Marília/Famema - Marília/SP.

Endereço para correspondência: Prof. Dr. Evandro Pereira Palacio, Serviço de Ortopedia e Traumatologia, Hospital de Clínicas da Faculdade de Medicina de Marília/Famema, Rua Monte Carmelo, 800 - 17519-030 Marília,SP ,Brasil. Tel.: (+55 14) 34021744. Fax: (+55 14) 3402-1701. E-mail: palacio@famema.br

Submetido em 21/11/2007 Versão final recebida em 01/04/2008 Aceito em 27/05/2008

\section{RESUMO}

Introdução e objetivos: Vários autores têm investigado a incidência de lesões no futebol, poucos têm-se preocupado com o tempo de afastamento dos atletas ou com os prejuízos financeiros oriundos de tal situação. O objetivo deste estudo foi confrontar, através de um estudo de coorte histórico, o tempo total de afastamento de atletas lesionados com as variáveis: idade, posição dos jogadores e tipo de lesão. Métodos: Foram analisados os jogadores de futebol profissional do Marília Atlético Clube que atuaram no Campeonato Brasileiro de Futebol - Série B, de 2003 a 2005. As informações foram coletadas através do acesso direto aos prontuários médicos e protocolo de coleta de dados, com um período de seguimento de 24 meses, sendo, posteriormente, realizada a análise estatística. Resultados: A idade dos jogadores variou de 18 a 35 anos (24,5 $\pm 4,4)$; os jogadores mais freqüentemente lesionados foram: atacantes (36,8\%), zagueiros (26,6\%), meias (20\%), laterais (10\%) e goleiros (6,6\%); as lesões mais comuns foram: musculares $(46,8 \%)$, ligamentares (26,6\%), ósseas $(16,6 \%)$ e meniscais (10\%); o tempo de afastamento nos atacantes variou de 10 a 240 dias (28 \pm 78,3), nos zagueiros de 20 a 120 dias $(26,5 \pm 33,9)$, nos meias de 10 a 180 dias $(18,5 \pm 66,3)$, nos laterais de 13 a 240 dias $(17 \pm 129,9)$ e nos goleiros de 35 a 60 dias $(47,5 \pm 17,6)$. Conclusão: A análise estatística mostrou não haver correlação entre a idade dos jogadores e o tempo total de afastamento dos mesmos $(p=0,31)$, não houve diferença significativa entre o tempo de afastamento nas diferentes posições dos jogadores dentro de campo $(p=0,49)$, houve diferença significativa entre o tempo total de afastamento e os diferentes tipos de lesão $(p<0,001)$ entre as variáveis: lesões meniscais e ósseas, lesões meniscais e musculares, lesões ligamentares e ósseas e, finalmente, lesões ligamentares e musculares.

Palavras-chave: medicina esportiva, lesões no futebol, epidemiologia do futebol, campeonato brasileiro de futebol.

\section{ABSTRACT}

Introduction and objectives: several authors have investigated the incidence of injuries occurred in outdoor soccer games. However, just a few are concerned about the athletes' healing period or the financial damage due to this no-activity period. The purpose of this study was to assess, through a cohort study, the injured athletes' recovery period, facing it to the variables: age, position in game and injure pattern. Methods: The medical reports of all professional soccer players of Marília Atlético Clube who were injured during the Brazilian Soccer Championship - $2^{\text {nd }}$ Division, from January/2003 to December/2005 were assessed, with a 24 months follow-up. Results: Athletes' mean age (SD) was 24.5 years (4.4 years); the most frequently injured athletes were: strikers (36.8), center-backs (26.6\%), center-midfielders (20\%), side-midfielders (10\%) and goalkeepers (6.6\%). The most frequent injuries were: muscular (46.8\%), ligament (26.6\%), bone (16.6\%) and meniscal (10\%); the strikers have shown a mean recovery period of 28 days (78.3 days), the center-backs 26.5 days (33.9), the center-midfielders 18.5 days (66.3), the side-midfielders 17 days (129.9) and the goalkeepers 47.5 days (17.6). Conclusion: Statistical analysis showed that there was no correlation between athletes' age and their healing period $(p=0.31)$; there was no statistical difference between the healing period among the five different athletes' positions $(p=0.49)$; there was statistical difference between the healing period and the injury pattern $(p<0.001)$ among the variables: meniscal and bone injuries, meniscal and muscular injuries, ligament and bone injuries and ligament and muscular injuries.

Keywords: sport medicine, soccer injuries, soccer epidemiology, brazilian soccer championship. 


\section{INTRODUÇÃO}

O futebol é a modalidade esportiva mais praticada e popular no mundo, com aproximadamente 400 milhões de adeptos em vários países, diferentes níveis sociais e faixas etárias ${ }^{(1)}$. Com a popularização cada vez maior da atividade futebolística, o número de lesões traumáticas graves também aumentou, uma vez que o futebol caracteriza-se pelo intenso contato físico, movimentos curtos, rápidos e não contínuos, tais como aceleração, desaceleração e mudanças abruptas de direção.

O excesso de jogos e treinamentos requer muito da capacidade física dos jogadores; qualidades como resistência, velocidade, agilidade e força os requisitos básicos para essa modalidade esportiva ${ }^{(2)}$. Kunze ${ }^{(3)}$ vai além, referindo que o futebol exige resistência, velocidade e força como princípios decisivos, mas também agilidade e flexibilidade, obrigando os jogadores a se expor a limites máximos de exaustão e, conseqüentemente, predispondo-os às lesões.

De acordo com Schenck, em se tratando de atletas, as lesões esportivas podem ser descritas como uma síndrome dolorosa que atue impedindo-os de desempenhar suas atividades esportivas, ou ainda, prejudicando sua performance ${ }^{(4)}$. Nos Estados Unidos, o Sistema Nacional de Registros de Lesões Esportivas (NAIRS) classifica as lesões esportivas, segundo o tempo de afastamento do atleta para recuperação, em três graus: lesões menores (de um a sete dias de afastamento), lesões moderadamente sérias (de oito a 21 dias de afastamento) e lesões sérias (acima de 21 dias de afastamento ou com lesões permanentes)(5).

O futebol é a maior causa de lesões em atletas no mundo e estas são responsáveis por 50 a 60\% de todas as lesões esportivas na Euro$\mathrm{pa}^{(6,7)}$. Dentre todos os traumas físicos tratados em hospitais europeus, de 3,5\% a 10\% são causados pelo futebol.

Do ponto de vista socioeconômico, deve-se salientar que, por vezes, os jogadores necessitam de cuidados médicos intensos e com diferentes períodos de reabilitação, algumas vezes com internação hospitalar ou no próprio departamento médico do clube. A ausência desses jogadores em suas atividades dentro de campo causa-Ihes grandes prejuízos financeiros e para suas carreiras, não mencionando alguns verdadeiros desastres financeiros para seus clubes, patrocinadores e agentes. Muitas vezes, o próprio atleta recusa-se a permanecer em tratamento ou mesmo receber a terapêutica conveniente, pois, no futebol, a pressão para o não afastamento e/ou a volta precoce do jogador ainda em tratamento são um fato comum(1).

A grande maioria dos estudos de metanálise existentes preconiza que a melhor forma de diminuir a incidência de agravos à saúde é por meio de prevenção com base em estudos de revisão sistemática e, principalmente, epidemiológicos ${ }^{(8)}$. Dessa forma, o objetivo deste estudo é analisar, através de um estudo de coorte histórico com seguimento de 24 meses, o tempo total de afastamento para recuperação de lesões dos jogadores de futebol profissional do Marília Atlético Clube que atuaram durante o Campeonato Brasileiros - Série B de 2003 a 2005, confrontando os resultados com as variáveis: idade, tipo de lesão e posição ocupada pelo jogador dentro de campo. Identificando, dessa forma, possíveis fatores que prolonguem a permanência do atleta em tratamento médico, em detrimento das carreiras pessoais e financeiras de jogadores, empresários e clubes.

\section{MÉTODOS}

\section{Amostra}

Inicialmente, foram selecionados para este estudo 90 jogadores do sexo masculino que atuaram no Marília Atlético Clube durante o Campeonato Brasileiro - Série B de 2003 a 2005. Para tanto, obteve-se consentimento para acesso e análise dos prontuários médicos dos atle- tas através de Termo de Consentimento Livre Esclarecido, assinado pelo vice-presidente do clube e também diretor do Departamento Médico, com a ressalva de que fossem preservadas as identificações dos atletas. Os critérios de inclusão foram: jogadores da categoria profissional com contrato assinado com o Marília Atlético Clube que foram afastados de suas atividades, devido a lesões traumato-ortopédicas, por, no mínimo, dez dias. Foram excluídos deste estudo os jogadores que apresentaram algum tipo de lesão traumato-ortopédica antes do início das temporadas, bem como os que se lesionaram em períodos de aquecimento físico ou exercícios resistidos (academia). Após a aplicação dos critérios de inclusão e exclusão, dos 90 jogadores inicialmente selecionados, foram incluídos neste estudo 30 atletas (tabela 1).

Os dados dos atletas incluídos foram coletados pelos autores, por acesso direto aos prontuários médicos, fazendo uso de Protocolo de Coleta de Dados (figura 1). Após um período de seguimento de 24 meses, os autores realizaram nova coleta de dados, acessando os prontuários médicos dos mesmos atletas, e, finalmente, tabulando os dados através do programa Excel" (Microsoft Office 2007).

Os diferentes tratamentos recebidos pelos atletas (cirurgia, fisioterapia e fortalecimento muscular) foram realizados sempre pela mesma equipe médica multidisciplinar do clube (médicos ortopedistas, fisioterapeutas e educadores físicos).

\section{PROTOCOLO DE COLETA DE DADOS}

01 - Atleta:

02 - Idade:

03 - Ano em que representou o clube:

$\begin{array}{lll}\text { ( ) } 2003 & \text { ( ) } 2004 & \text { ( ) } 2005\end{array}$

04 - Posição:

( )Atacante ( )Goleiro ( )Lateral ( )Meia ( )Zagueiro

05 - Situação contratual

( )Contrato assinado ( )Período de experiência

( ) Em teste (categoria de base) ( ) Em préstimo

06 - Lesões anteriores e datas:

07 - Tipos de lesão:

( ) Lesão óssea ( )Lesão ligamentar ( )Lesão Meniscal ( ) Lesão Muscular ( ) Outros

08 - Data da nova lesão:

09 - Breve história da lesão:

10 - Exames realizados:

11 - Resultado dos exames:

12 - Tratamento realizado:

13 - Data do retorno às atividades:

14 - Condições de retorno

( ) Curado ( )Melhorado ( ) Com restrições (tipo )

15 - Médico(s)

responsável(eis):

Figura 1. Formulário para registro das lesões. 
Tabela 1. Dados gerais dos atletas incluídos no estudo

\begin{tabular}{|c|c|c|c|c|c|c|c|}
\hline $\begin{array}{l}\text { No. de } \\
\text { ordem }\end{array}$ & Nome & Idade & Ano & Posição & Tipo de lesão & Tratamento & $\begin{array}{c}\text { Período de } \\
\text { afastamento (dias) }\end{array}$ \\
\hline 1 & R.S.S. & 26 & 2003 & Atacante & Lesão dos ligamentos talofibular anterior D. + calcaneofibular D. & Cirurgia & 120 \\
\hline 2 & A. A.S. & 20 & 2003 & Atacante & Distensão (grau II) do reto da coxa D. & Repouso, fisioterapia & 21 \\
\hline 3 & E. D.S. L. & 22 & 2003 & Atacante & Lesão (grau II) ligamento colateral medial E. & $\begin{array}{l}\text { Repouso, imobilização e } \\
\text { fisioterapia }\end{array}$ & 35 \\
\hline 4 & M. F. D. R. & 28 & 2003 & Atacante & Distensão (grau II) do semitendíneo D. & Repouso e fisioterapia & 15 \\
\hline 5 & S.T. O. & 30 & 2003 & Atacante & Lesão ligamento cruzado anterior D. & Cirurgia & 240 \\
\hline 6 & A.S.G. & 21 & 2003 & Meia & Lesão ligamento colateral medial D. + menisco medial D. & Cirurgia & 180 \\
\hline 7 & A. S. & 27 & 2003 & Meia & Distensão (grau I) do iliopsoas D. & Repouso, fisioterapia & 10 \\
\hline 8 & A. A.S. & 30 & 2003 & Zagueiro & Distensão (grau II) do gastrocnêmio D. & Repouso, fisioterapia & 28 \\
\hline 9 & A. F. & 30 & 2003 & Zagueiro & Distensão (grau II) do adutor magno E. & Repouso, fisioterapia & 28 \\
\hline 10 & H. H. B. & 22 & 2003 & Zagueiro & Lesão do ligamento colateral medial D. + menisco medial D. & Cirurgia & 120 \\
\hline 11 & A.M. S.F. & 26 & 2003 & Lateral & Lesão do ligamento cruzado anterior + menisco medial D. & Cirurgia & 240 \\
\hline 12 & A.M.S. & 25 & 2003 & Atacante & Distensão (grau II) do reto da coxa D. & Repouso e fisioterapia & 21 \\
\hline 13 & C.M.S. & 35 & 2003 & Atacante & Lesão do ligamento colateral medial D. & $\begin{array}{l}\text { Repouso, imobilização e } \\
\text { fisioterapia }\end{array}$ & 30 \\
\hline 14 & R.W.C. & 21 & 2004 & Atacante & Distensão (grau II) do gastrocnêmio D. & Repouso e fisioterapia & 24 \\
\hline 15 & O. J. M. J. & 22 & 2004 & Meia & Lesão do ligamento colateral medial D. & Repouso e fisioterapia & 30 \\
\hline 16 & C. L.R. & 23 & 2004 & Meia & Lesão (grau II) do iliopsoas D. & Repouso e fisioterapia & 19 \\
\hline 17 & E.F.V.S. & 20 & 2004 & Zagueiro & Lesão crônica-agudizada do gastrocnêmio D. & Repouso e fisioterapia & 25 \\
\hline 18 & H. H. B. & 23 & 2004 & Zagueiro & Distensão (grau II) do adutor magno D. & Fisioterapia e RPG & 28 \\
\hline 19 & W. F. A. & 35 & 2004 & Zagueiro & Distensão (grau II) do quadríceps D. & Repouso, fisioterapia e RPG & 21 \\
\hline 20 & D.D.S.S. & 20 & 2004 & Lateral & Fratura do $5^{\circ}$ artelho $\mathrm{E}$. & Imobilização + repouso & 13 \\
\hline 21 & B. C.P. & 24 & 2004 & Goleiro & Fratura do processo estilóide do rádio $D$. & Imobilização e fisioterapia & 35 \\
\hline 22 & H. G.S. & 23 & 2005 & Atacante & Lesão do ligamento cruzado anterior D. & Cirurgia & 180 \\
\hline 23 & R.W.C. & 22 & 2005 & Atacante & Lesão do ligamento colateral medial D. & Repouso e fisioterapia & 28 \\
\hline 24 & M. F.M.S. & 25 & 2005 & Atacante & Distensão (grau II) do reto da coxa D. & Repouso, fisioterapia e RPG & 10 \\
\hline 25 & C.F.C. & 19 & 2005 & Meia & Fratura incompleta do cuneiforme medial em pé E. & Imobilização e repouso & 18 \\
\hline 26 & J.L.S. & 28 & 2005 & Meia & Distensão (grau II) do adutor magno D. & Repouso, fisioterapia e RPG & 15 \\
\hline 27 & E.F.V.S. & 21 & 2005 & Zagueiro & Distensão (grau II) do bíceps da coxa D. & Repouso, fisioterapia e RPG & 21 \\
\hline 28 & J.F.G.M. & 22 & 2005 & Zagueiro & Fratura cominutiva da falange média do $3^{\circ}$ dedo mão $\mathrm{E}$. & Cirurgia & 20 \\
\hline 29 & A. F.M. & 18 & 2005 & Lateral & Fratura da falange proximal do hálux E. & Imobilização e repouso & 17 \\
\hline 30 & A.G.S. & 29 & 2005 & Goleiro & Luxação glenoumeral + lesão labrum & Cirurgia & 60 \\
\hline
\end{tabular}

Fonte: Departamento médico Marilia Atlético Clube

Para a realização da análise estatística foi utilizado o programa SigmaStat ${ }^{\text {TM }}$ versão 3.11 (Systat Software, Inc.). Foram utilizados testes estatísticos não-paramétricos e descritivos, sempre adotando o nível de significância de 0,05.

\section{RESULTADOS}

A idade dos jogadores variou de 18 a 35 anos $(24,5 \pm 4,4)$; as lesões mais comuns foram: 14 musculares (46,8\%), oito ligamentares (26,6\%), cinco ósseas $(16,6 \%)$ e três meniscais (10\%); o tempo de afastamento nos atacantes variou de 10 a 240 dias $(28 \pm 78,3)$, nos zagueiros de 20 a 120 dias $(26,5 \pm 33,9)$, nos meias de 10 a 180 dias $(18,5 \pm 66,3)$, nos laterais de 13 a 240 dias $(17 \pm 129,9)$ e nos goleiros de 35 a 60 dias $(47,5 \pm 17,6)$. O tratamento prestado aos atletas lesionados dividiu-se em 22 conservadores (73,3\%) e oito cirúrgicos (26,6\%).

Quando comparamos o número de jogadores lesionados e as posições dos mesmos, verificamos não haver diferença estatisticamente significativa entre os atacantes $(36,8 \%)$, zagueiros $(26,6 \%)$ e meio-campistas (20\%) $(p=0,06)$; contudo, encontramos tendência à diferença quando os comparamos às posições de laterais (10\%) e goleiros $(6,6 \%)(p=0,05)$.

Com referência à possível relação entre as idades dos jogadores lesionados e o tempo em que cada um deles permaneceu afastado em tratamento médico, o teste de correlação de Spearman apresentou um valor de $p=0,31$, evidenciando não haver correlação entre o tempo de afastamento dos jogadores e a idade dos mesmos (figura 2).

Se levarmos em consideração o tempo de afastamento e a posição de cada jogador (atacante, meia, zagueiro, lateral e goleiro), o teste não-paramétrico de Kruskal-Wallis apresentou um valor de $p$ de 0,49, não havendo, portanto, diferença estatisticamente significativa entre estas duas variáveis (figura 3).

Diferentemente dos resultados anteriores, ao analisarmos o tempo de afastamento dos atletas e o tipo de lesão sofrida pelos mesmos, o teste não-paramétrico de Kruskal-Wallis mostra um valor de $p<0,001$, havendo, portanto, diferença estatisticamente significativa entre o tem- 


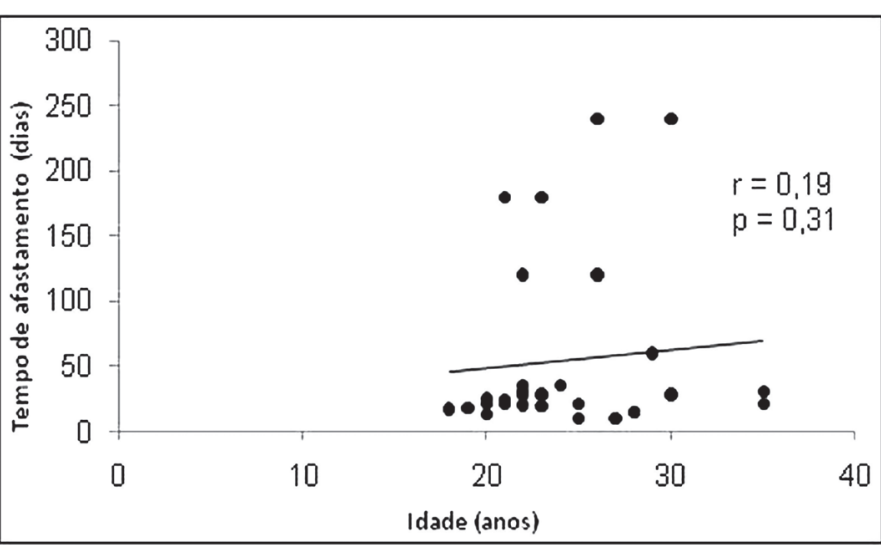

Figura 2. Gráfico de regressão mostrando não haver correlação entre o tempo de afastamento dos jogadores e a idade dos mesmos.

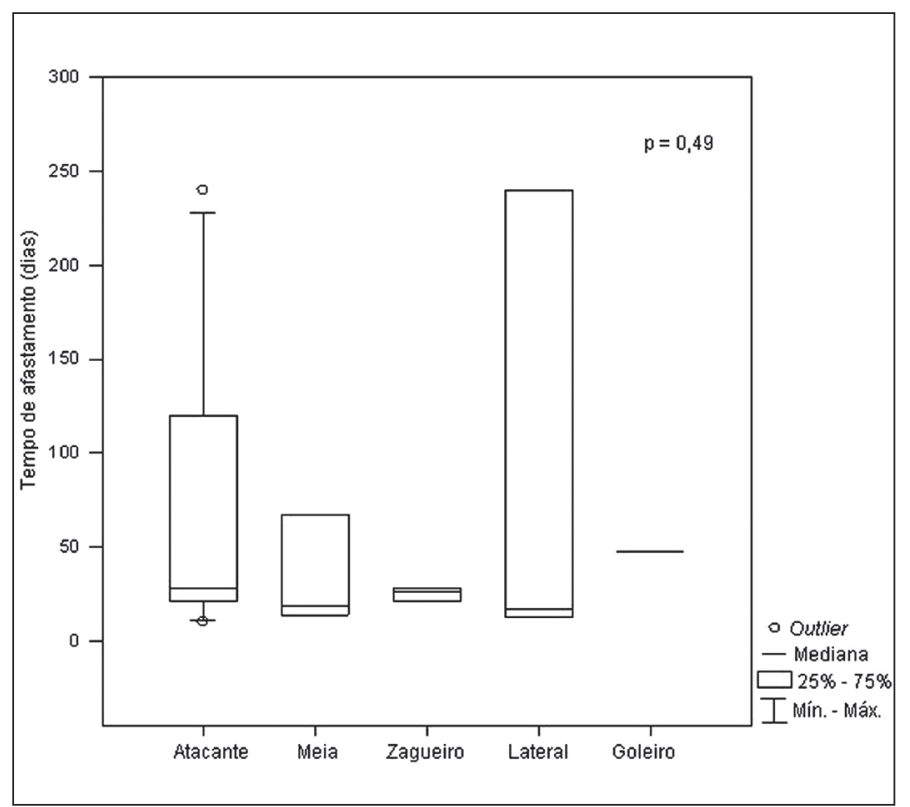

Figura 3. Gráfico tipo box plot com os valores centrais e valores mínimos e máximos, em relação ao tempo de afastamento dos jogadores e a posição dos mesmos.

po de afastamento e o tipo das lesões (meniscal, ligamentar, muscular e óssea) (figura 4). Utilizando ainda o teste de Dunn para comparações múltiplas (tabela 2), o mesmo demonstra diferença estatisticamente significativa $(p<0,05)$ entre as seguintes variáveis:

- Lesões meniscais e lesões ósseas: Sim

- Lesões meniscais e lesões musculares: Sim

- Lesões meniscais e lesões ligamentares: Não

- Lesões ligamentares e lesões ósseas: Sim

- Lesões ligamentares e lesões musculares: Sim

- Lesões musculares e lesões ósseas: Não

Tabela 2. Teste de comparações múltiplas de Dunn $(p<0,05)$

\begin{tabular}{l|c|c|c|c}
\hline Tipo de lesão & $\mathbf{n}$ & Mediana & DP & Conjunto * \\
\hline Meniscal & 3 & 180 & 60 & $\mathrm{~A}$ \\
\hline Óssea & 5 & 18 & 8,4 & $\mathrm{~B}$ \\
\hline Ligamentar & 8 & 47,5 & 81,4 & $\mathrm{~A}$ \\
\hline Muscular & 14 & 21 & 6,1 & $\mathrm{~B}$ \\
\hline
\end{tabular}

* Medianas seguidas de letras iguais indicam que os conjuntos não diferem estatisticamente DP: desvio padrão

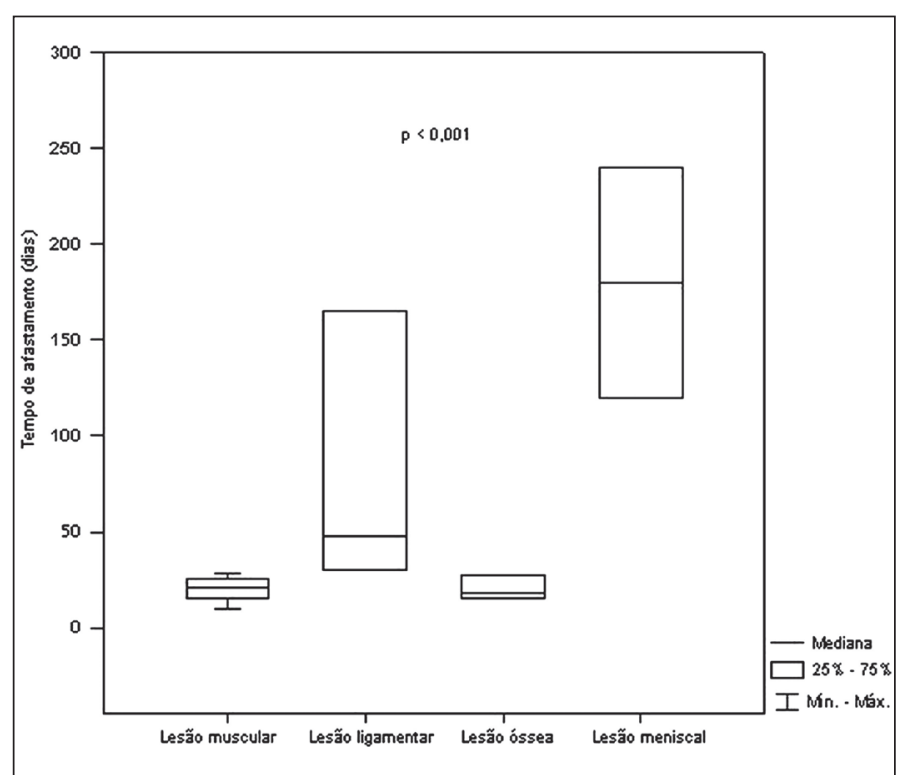

Figura 4. Gráfico tipo box plot com os valores centrais e valores mínimos e máximos, em relação ao tempo de afastamento dos jogadores e o tipo de lesão.

\section{DISCUSSÃO}

É fato notório, atualmente, que atletas fisicamente lesionados e "internados" nos diversos departamentos médicos dos clubes, por períodos variáveis, significam prejuízo financeiro para todos. O futebol e seus representantes, como qualquer outra empresa, além de resultados públicos positivos, visam, principalmente, aumento de receita. Através deste estudo de coorte histórico, procurou-se correlacionar o tempo total de afastamento dos jogadores com outras três variáveis: idade, o tipo de lesão e a posição dos atletas dentro de campo, almejando identificar fatores que causem o prolongamento do tempo de recuperação e procurando meios de minimizá-los.

No que se refere à freqüência de lesões nas cinco posições dos jogadores (atacantes, zagueiros, meias, laterais e goleiros), nossos resultados concordam com os de diversos autores ${ }^{(1,9-11)}$, uma vez que, neste estudo, as maiores incidências de lesões ocorreram nos atacantes (36,8\%), zagueiros (26,6\%) e meias (20\%). Esse resultado pode ser explicado se levarmos em consideração o novo estilo do futebol mundial: a força, a velocidade e, principalmente, as mudanças de direção e aceleração, em níveis extremos, predispõem tanto o ataque quanto a defesa a maiores chances de se lesionar ${ }^{(12)}$. Esses atletas, atacantes e zagueiros, são mais exigidos fisicamente que os demais, realizando movimentos rotacionais em excesso e percorrendo maiores distâncias em velocidade máxima, aumentando, ainda mais, os índices de contusões ${ }^{(1,9-11,13)}$.

Alguns estudos mostram uma associação direta entre idade e freqüência de lesões em atletas profissionais de futebol. Nossos dados mais uma vez concordam com os de outros autores ${ }^{(11)}$, onde se verifica que a maior parte das lesões (60\%) ocorreu em jogadores com idade entre 20 e 25 anos.

Verificamos, assim como em outros estudos ${ }^{(1,11,14)}$, que as lesões mais freqüentes são as musculares $(46,8 \%)$ e, ainda analisando o tipo de lesão, concluímos que a menos comum é a meniscal; por outro lado, é a que mais tempo mantém os atletas longe de suas atividades profissionais, com média de 180 dias ( \pm 60 dias).

De acordo com o tempo de afastamento, neste estudo os dados encontrados variaram de 10 a 240 dias. A grande amplitude (230 dias) deve-se ao fato de termos incluído, no mesmo estudo, injúrias leves como algumas contusões musculares e lesões graves como as menis- 
cais e as do ligamento cruzado anterior, o que resultou em períodos de reabilitação que se arrastaram, em alguns casos, para além de seis meses $^{(15-19)}$

A análise estatística mostrou que não existe correlação entre a idade dos atletas e o tempo de reabilitação $(p=0,31)$. Dessa maneira, pode-se inferir que os elevados períodos de afastamento de alguns atletas deram-se unicamente devido à natureza de suas lesões, não se relacionando com a idade dos mesmos ${ }^{(6)}$. Da mesma maneira, os testes estatísticos mostraram não existir diferença significativa entre o tempo de afastamento e a posição ocupada pelos jogadores dentro de campo $(p=0,49)$; assim, verificamos que atacantes, zagueiros, laterais, meias e goleiros apresentam períodos de reabilitação semelhantes.

Quanto ao tempo de afastamento dos jogadores e o tipo de lesão que sofreram, encontrou-se diferença significativa $(p<0,001)$ entre algumas variáveis. O teste de comparações múltiplas de Dunn identificou haver diferença significativa $(p<0,05)$ entre as lesões meniscais e ósseas, lesões meniscais e musculares, lesões ligamentares e ósseas e entre lesões ligamentares e musculares. A vasta literatura médica especializada ${ }^{(19-22)}$ confirma tais dados, uma vez que, dentre as variáveis em estudo, as lesões meniscais são as que mais tempo demandam para sua total reabilitação, principalmente quando associadas a outras lesões. As lesões ligamentares vêm logo a seguir das meniscais e, finalmente, as musculares ${ }^{(1,6,12,24-26)}$. Neste estudo, os dados referentes às lesões ósseas devem ser observados com cautela, uma vez que não encontramos atletas que apresentassem fraturas graves (ossos longos, fraturas expostas, etc.). Os poucos casos de fraturas aqui encontrados referem-se a fraturas simples de processos ósseos ou ainda ossos curtos, que se resolvem, sem complicações, como uso de talas ou órteses e com poucos dias de tratamento ${ }^{(27,28)}$. Tal fato certamente interferiu nos resultados, classificando as lesões ósseas como as que menos tempo mantêm o atleta afastado de suas atividades, o que não deixa de ser uma verdade, porém, somente para este estudo em particular.

\section{CONCLUSÃO}

Os dados apontados por este estudo, após análises estatísticas especializadas, permitiram algumas conclusões únicas:

1) Não há correlação entre o tempo total de afastamento dos jogadores para reabilitação e a idade dos mesmos. Atletas mais jovens e mais velhos recuperam-se de suas contusões em tempos semeIhantes.

2) Não há diferença entre o tempo total de afastamento dos atletas para reabilitação e a posição em que estes jogam. Alguns jogadores, como atacantes, zagueiros e meias, tendem a se contundir com maior freqüência quando comparados com os laterais e goleiros, porém, o tempo de convalescência é semelhante para todas as cinco posições de atuação estudadas.

3) Existe diferença entre o tempo total de afastamento dos atletas e o tipo de lesão que os mesmos apresentam (meniscal, ligamentar, muscular ou óssea). O ideal seria que os jogadores fossem submetidos a programas de treinamento específicos (principalmente com propriocepção, fortalecimento muscular, alongamentos e outros), com o intuito exclusivo de diminuir a freqüência de lesões.

Todos os autores declararam não haver qualquer potencial conflito de interesses referente a este artigo.

\section{REFERÊNCIAS BIBLIOGRÁFICAS}

1. Cohen M, Abdalla RJ, Ejnisman B, Amaro JT. Lesões ortopédicas no futebol. Rev Bras Ortop. 1997;32(12):940-4

2. Garcia CM, Muiño ET, Teleña AP. La preparación física en el fútbol. Madrid: Editorial Augusto, 1983.

3. Kunze A. Futebol. Lisboa: Desporto, 1987.

4. Schenck RC Jr. Lesão esportiva e a reposta dos tecidos à lesão física. Medicina Esportiva e Treinamento Físico Atlético, 2003. P. 128

5. Vital R, Silva HGPV, Sousa RPA, Nascimento RB, Rocha EA, Miranda HF, et al. Lesões traumato-ortopédicas nos atletas paraolímpicos. Rev Bras Med Esporte. 2007;13(3):165-8.

6. Keller CS, Noyes FR, Buncher CR. The medical aspects of soccer injury epidemiology. Am J Sports Med. 1987;15(3):230-7

7. Silva AA, Dória DD, Morais GA, Prota RVM, Mendes VB, Lacerda AC, et al. Fisioterapia esportiva: prevenção e reabilitação de lesões em atletas do América Futebol Clube. Anais do $8^{\circ}$ Encontro de Extensão da UFMG. Belo Horizonte: 2005.

8. Sackett DL, Richardson WS, Rosenberg W, Haynes RD. Evidence-Based Medicine: how to practice and teach. London: Churchill Livingstone; 1997.

9. Pedrinelli A. Incidência de lesões traumáticas em atletas de futebol. Dissertação de mestrado, Faculdade de Medicina da Universidade de São Paulo, 1994.

10. Stewien ETM, Camargo OPA. Ocorrência de entorse e lesões do joelho em jogadores de futebol da cidade de Manaus, Amazonas. Acta Ortop Bras. 2005;13(3):141-6.

11. Raymundo JLP, Reckers $L$, Locks R, Silva L, Hallal PC. Perfil das lesões e evolução da capacidade física em atletas profissionais de futebol durante uma temporada. Rev Bras Ortop. 2005;40(6):341-8.

12. Faria LF, Paiva VH. Incidência de lesões em jogadores de futebol profissional do Uberaba Sport Clube no campeonato mineiro módulo II 2005. Anais do IV Workshop em Fisiologia do Exercício da UFSCar. São Carlos: 2005.

13. Gould III JA. Fisioterapia na ortopedia e na medicina do esporte. São Paulo: Manole, 1993.
14. Arnason A, Gudmundsson A, Dahl HA, Jóhannsson E. Soccer injuries in Iceland. Scand J Med Sci Sports. 1996;6(1):40-5

15. Bjordal JM, Arnøy F, Hannestad B, Strand T. Epidemilogy of anterior cruciate ligament injuries in soccer. Am J Sports Med. 1997;25(3):341-5.

16. Lopes AS, Kattan R, Costa S, Moura CE. Estudo clínico e classificação das lesões musculares. Rev Bras Ortop. 1993;28(10):707-17.

17. McMaster WC, Walter M. Injuries in soccer. Am J Sports Med. 1978;6(6):354-7.

18. Buceta JM. Psicologia y lesiones deportivas: prevención y recuperación. Madrid: Dykinson, 1996.

19. Amatuzi MM, Hernandez AJ, Albuquerque RFM. Lesões menisco-ligamentares do joelho. In: Herbert S, Xavier R. Ortopedia e traumatologia: princípios e prática. Porto Alegre: Artmed, 1998;672-82.

20. Palma IM. Lesões do ligamento cruzado anterior do joelho. In: SBOT. Programa de atualização em traumatologia e ortopedia (PROATO). Porto Alegre: Artmed/Panamericana. 2005;111-35.

21. Thiele E, Carvalho H, Pedrinelli A, Carvalho D. Lesão muscular no esporte. In: SBOT. Programa de atualização em traumatologia e ortopedia (PROATO). Porto Alegre: Artmed/Panamericana, 2006;83-134

22. Andrade MAP, Grossi GCX. Instabilidade femoropatelar. In: SBOT. Programa de atualização em traumatologia e ortopedia (PROATO). Porto Alegre: Artmed/Panamericana, 2006;49-85.

23. Carazzato JG. Manual de medicina do esporte. São Paulo: Laboratório Pfizer. 1993;4-41.

24. Ladeira CE. Incidência de lesões no futebol: um estudo prospectivo com jogadores masculinos adultos amadores canadenses. Rev Bras Fisiot. 1999;4:39-47.

25. Naves J. Medicina Del deporte y accidentes desportivos. Barcelona: Editores Salvat, 1977.

26. Silva PRS. O papel do fisiologista desportivo no futebol - Para quê? e Por quê? Reabilitar. 2001;13:30-5.

27. Cooney III WP, Linscheid RL, Dobyns JH. Fraturas e luxações do punho. In: Rockwood Jr CA, Green AP, Bucholz RW. Fraturas em adultos. São Paulo: Manole, 1993;1:553-91.

28. Heckman JD. Fraturas e luxaçōes do pé. In: Rockwood Jr CA, Green AP, Bucholz RW. Fraturas em adultos. São Paulo: Manole, 1993; 2:2111-41. 